\title{
Recognition of Elementary Upper Limb Movements in an Activity of Daily Living using Data from Wrist Mounted Accelerometers
}

\author{
Dwaipayan Biswas, Andy Cranny, Nayaab Gupta, \\ Koushik Maharatna \\ Faculty of Physical Sciences and Engineering \\ University of Southampton \\ Highfield, Southampton, SO17 1BJ UK \\ Email: \{db9g10, awc,ng2g10, km3\}@ecs.soton.ac.uk
}

\author{
Steffen Ortmann \\ IHP - Innovations for High Performance Microelectronics \\ Leibniz-Institut für Innovative Mikroelektronik \\ Frankfurt (Oder), Germany \\ Email: ortmann@ihp-microelectronics.com
}

\begin{abstract}
In this paper we present a methodology as a proofof-concept for recognizing fundamental movements of the human arm (extension, flexion and rotation of the forearm) involved in 'making-a-cup-of-tea', typical of an activity of daily-living (ADL). The movements are initially performed in a controlled environment as part of a training phase and the data are grouped into three clusters using $k$-means clustering. Movements performed during ADL, forming part of the testing phase, are associated with each cluster label using a minimum distance classifier in a multi-dimensional feature space, comprising of features selected from a ranked set of 30 features, using Euclidean and Mahalonobis distance as the metric. Experiments were performed with four healthy subjects and our results show that the proposed methodology can detect the three movements with an overall average accuracy of $88 \%$ across all subjects and arm movement types using Euclidean distance classifier.
\end{abstract}

Keywords-accelerometer; activities of daily living (ADL); activity recognition; clustering; minimum distance classifier; movement classification.

\section{INTRODUCTION}

Motion tracking of the human body has been used in a wide range of applications such as home-based remote health monitoring [1-2], human computer interaction [3-4] and sports coaching [5-6] using various sensing technologies including: mechanical tracking, optical systems, radio-frequency identification (RFID) [7], low-cost body-worn inertial sensors [8-9], and fusion of vision-based and inertial sensor based approaches [10]. Nowadays, body-worn inertial sensors are low cost and are therefore a popular technique to track movements effectively. The sensor data collected is used to train an activity model for recognizing the activities from new observation data [11]. The considerable amount of variability inherent in movement patterns of each subject, poses a significant challenge towards recognition of activities during unconstrained daily living from sensor data without manual intervention or a-priori knowledge [12].

In this paper, we report on a systematic exploration to recognize three fundamental movements of the upper limb that are generally associated with activities of daily living, using

This work was supported by the European Union under the $7^{\text {th }}$ Framework Programme, grant agreement \#288692, StrokeBack. data collected only from a wrist-worn, wireless tri-axial accelerometer. The motivation behind this work was to detect the occurrence of these specific arm movements in a real world scenario (i.e. unconstrained environment) using the minimal number of sensors, as opposed to detecting gross static or dynamic activities and postures like standing, sitting, running, cycling, brushing teeth $[1,6,9]$, etc., using a combination of inertial, magnetic and optical sensors. The detection and classification of particular arm movements (e.g. prescribed exercises) during daily activities, over time can provide a measure of rehabilitation progress in several remote health monitoring applications. It will be particularly useful in the monitoring of arm rehabilitation in pathologies associated with neurodegenerative diseases such as stroke or cerebral palsy. The specific arm movements considered were: (1) reach and retrieve object, (2) lift cup to mouth and (3) performing pouring/(un)locking action, all of which involve rotations of the forearm about various axes. Continuous monitoring of activities in an unconstrained scenario involves data segmentation and activity recognition which are in practice interrelated but are individually two separate research problems owing to the possible qualitative non-uniqueness of an activity pattern exhibited by an individual subject and due to interperson variability. Here, we concentrate only on the activity recognition part as a proof-of-concept methodology.

The fundamental concept is to formulate a set of clusters each uniquely capturing the essence of multi-dimensional features (selected from a ranked set of 30 features) of a particular type of movement (three clusters corresponding to the three chosen movements in our case) from a feature set generated by a set of person-centric training data collected in the laboratory (training phase) and then applying a pattern recognition technique associating multidimensional feature data collected while performing a real world activity in an unconstrained scenario (testing phase) to these predefined cluster labels for recognizing the movement. We used the regularized Mahalonobis distance based $k$-means clustering technique to form the pre-defined clusters on the training data and use an Euclidean and Mahalonobis distance based minimum distance classifier for assigning the test data to the formed clusters. We adopted a personalized approach to pattern 
recognition, thereby formulating the clusters from personcentric training data. This approach was adopted in view of the large degree of inter-person variability expected amongst the sample population. Furthermore, this would be beneficial when applied to monitoring individual patients who demonstrate differences in levels of impairment depending on their stage of rehabilitation. Experiments performed with four healthy subjects show that the proposed method can recognize the three movements with an overall average accuracy of $88 \%$ across all subjects and arm movement types.

The remainder of the paper is structured as follows. In Section II we provide a background to the state-of-the-art techniques for activity recognition, relating prior work to our methodology, and in Section III we describe the experiment protocol. The data processing techniques and the results are analyzed in Section IV and V respectively. Finally, a discussion is presented in Section VI.

\section{BACKGROUND}

Activity recognition of gross movements and postures using inertial sensors is generally analyzed using statistical signal processing involving the primary steps of feature extraction, feature selection and classification $[1,6,8,9,13]$. Different machine learning techniques have been used for such movement recognition, e.g. Support Vector Machines (SVM) [14-15], Decision Trees (DT) [8, 13], Naive Bayes (NB) [13], Multi-Layer Perceptron (MLP) [16], Artificial Neural Networks (ANN) [8], Hidden Markov Models (HMM) [17], or a combination of these techniques [10]. Instance-based classification algorithms have been used successfully over conventional algorithms to classify data from inertial sensors but suffer from high memory usage and long classification times [18].

To the best of our knowledge, very little has been reported on recognizing specific upper limb movements in an unconstrained scenario, which is an important aspect for assessing rehabilitation of impaired limb functionality such as in stroke. Clustering techniques have been extensively used in diverse fields such as medicine (EEG, Functional MRI) psychology, geography (mining, earthquake detection) or marketing (seasonality patterns in finance and retail) and has produced successful results across disciplines and can be conveniently deployed with limited resources (memory and CPU) [19]. We extend it further to activity recognition involving the upper limb by using the widely popular $k$-means partitional clustering algorithm.

The accuracy of any movement recognition technique is dependent on the system components and requirements, covering areas such as: type of activities, number of activities, type of sensors, number of sensors, placement of sensors [16], level of data fusion, and most importantly the classification methodology adopted. Further, there is a need for subject specific training especially for tracking activities that are susceptible to individual and temporal variation [20]. Recognition strategies generally follow one of three themes. Firstly, using only data collected under controlled conditions (i.e. in the laboratory) for training as well as testing, which results in high accuracies [8]. Secondly, using both controlled and un-controlled data for both training and testing, which results in reasonably high accuracies [6, 20]. Finally, using controlled data for training and only un-controlled data for testing, which generally results in lower accuracies $[6,20]$.

It has been suggested that to increase the levels of accuracy there is a need to train the classifier with un-controlled data as well as controlled (training) data [6]. In this work we concentrated on using only the controlled data for training and un-controlled data for testing to explore the levels of recognition accuracy applicable in the field of rehabilitation in home settings, where a subject is instructed to follow a particular exercise regime to be performed in a controlled environment (clinic or home) and is later monitored to track the occurrence of the specific exercises while they perform their daily activities involving the impaired arm, facilitating rehabilitation progress.

\section{EXPERIMENTAL PROTOCOL}

A Shimmer 9DoF wireless kinematic sensor module comprising mutually orthogonal tri-axial accelerometers, rate gyroscopes and magnetometers, was used as the sensing platform [21]. For our experiments we only use the tri-axial accelerometer with $\pm 1.5 \mathrm{~g}$ range selected and exclude the magnetometer since it can be affected by the presence of ferromagnetic materials which are expected to be present in the natural environment [22]. We also leave out the gyroscope in view of using a minimal number of sensors aimed at reducing the amount of data processing involved. The dorsal side of the forearm proximal to the wrist on the dominant arm was chosen as the sensing position since it was likely to produce significant sensor responses to the arm movements being investigated. The dorsal side was in contact with the XY plane of the sensor with the $\mathrm{X}$-axis pointing towards the hand and the Z-axis pointing away from the dorsal aspect. Sensor data was collected at a rate of $50 \mathrm{~Hz}$, deemed sufficient for assessing habitual limb movement, which is on the higher side compared to assessing holistic activity as in $[13,16]$.

We focused on three elementary types of arm movement (actions):

- $\quad$ Action $A$ - reach and retrieve an object.

- $\quad$ Action B-lift forearm to mouth.

- $\quad$ Action $C$ - perform pouring or (un)locking action.

In principle, these elementary movements constitute a significant proportion of the complex movements performed with the upper limb in daily life and also resemble three of the tasks in the standard Wolf Motor Function Test (WMFT); an established clinical assessment method for quantifying upper extremity motor ability [23-25]. In this investigation, experiments were performed with four healthy subjects (age range 24 to 40, male, all right arm dominant) within an open laboratory with an attached kitchen at University of Southampton. To generate the training phase data for the target cluster formation all four participants performed 240 trials of Action A, 120 trials of Action B and 120 trials of Action $C$, separated into groups of five repetitions, with each group of trials being separated by approximately three minutes. This was done to avoid unrepresentative data due to 
fatigue and to minimize the effects of unconscious selflearning of the activities. This data collection facilitates to have a large training set offering greater convenience for accurate recognition [20] since the cluster formulation on the training data inherently captures the person-centric nature of movement patterns. There were more number of trials for Action $A$ since it is a generic movement performed more frequently in our daily lives as is further evident from Table I.

We designed an activity-list (cf. Table I) which emulated the process of 'making-a-cup-of-tea', a common activity performed in daily life, having repeated occurrences of the three elementary types of arm movement (actions). The activity list in our experiment protocol comprises 20 individual activities including 10 occurrences of Action A, and 5 each of Action $B$ and Action $C$. There were no restrictions on the various physical factors of the experiment such as the seating position or standing position with respect to the kitchen surface or the time required to complete the actions. The experiment was unconstrained in this manner to ensure a wider range of variability in the data paving the way for a robust arm movement recognition system which will produce acceptable levels of accuracy in a real world application.

TABLE I. USE CASE ACTIVITY LIST - 'MAKING-A-CUP-OF-TEA'

\begin{tabular}{|l|c|}
\hline \multicolumn{1}{|c|}{ Activity } & Action \\
\hline 1. Fetch cup from desk & $\mathrm{A}$ \\
\hline 2. Place cup on kitchen surface & $\mathrm{A}$ \\
\hline 3. Fetch kettle & $\mathrm{A}$ \\
\hline 4. Pour out extra water from kettle & $\mathrm{C}$ \\
\hline 5. Put kettle onto charging point & $\mathrm{A}$ \\
\hline 6. Reach out for the power switch on the wall & $\mathrm{A}$ \\
\hline 7. Drink a glass of water while waiting for kettle to boil & $\mathrm{B}$ \\
\hline 8. Reach out to switch off the kettle & $\mathrm{A}$ \\
\hline 9. Pour hot water from the kettle in to cup & $\mathrm{C}$ \\
\hline 10. Fetch milk from the shelf & $\mathrm{A}$ \\
\hline 11. Pour milk into cup & $\mathrm{C}$ \\
\hline 12. Put the bottle of milk back on shelf & $\mathrm{A}$ \\
\hline 13. Fetch cup from kitchen surface & $\mathrm{A}$ \\
\hline 14. Have a sip and taste the drink & B \\
\hline 15. Have another sip while walking back to desk & B \\
\hline 16. Unlock drawer & C \\
\hline 17. Retrieve biscuits from drawer & A \\
\hline 18. Eat a biscuit & B \\
\hline 19. Lock drawer & B \\
\hline 20. Have a drink & B \\
\hline
\end{tabular}

On a separate day, participants were recalled and requested to perform four repetitions of the activity-list in Table 1, at a comfortable speed in a kitchen, with a 10 minute rest period between repetitions. The activity-list was prepared to facilitate the evaluation of the recognition methodology under seminaturalistic conditions. The start and stop time of the activities were noted down by the researcher observing them as they performed their designated tasks naturally. The corresponding data collected was segmented using the annotations from the researcher and used for the testing phase. Since our focus was mainly on recognizing the occurrence of these elementary movements, we did not implement an automated segmentation method. We, therefore have 1440 vectors comprising of samples of acceleration values $\{[(240$ of $A)+(120$ of $B)+$ $(120$ of $C)] \times 3$-axis accelerometer $\}$ from the training phase and 240 vectors $\{[(10$ of $A)+(5$ of $B)+(5$ of $C)] \times 4$ trials $\times$ 3 -axis accelerometer from the testing phase for each subject.

\section{DATA PROCESSING}

The primary steps involved in our data processing for each subject's data is illustrated in Fig. 1 and described in detail in the following sections.

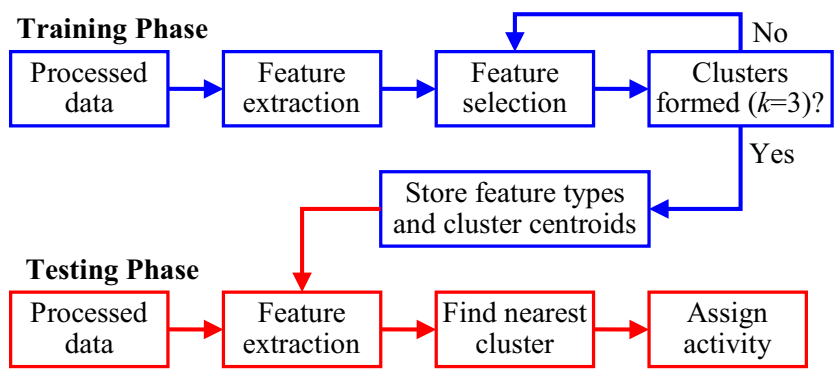

Fig. 1. Basic stages of data processing.

\section{A. Acquisition and Pre-processing}

The tri-axial accelerometer located on the wrist transmits data along with a time stamp to a host computer using the Bluetooth wireless transmission protocol and each activity performed by a subject is marked to record the start and end of the movement for all trials performed during the training and testing phases. The raw sensor data is band-pass filtered with a 3rd order Butterworth filter having cut-off frequencies of 0.1 $\mathrm{Hz}$ and $12 \mathrm{~Hz}$ to respectively attenuate the low frequency artifacts and high frequency noise components introduced in the data due to physical effects such as drift [13].

\section{B. Feature Extraction}

Typical feature sets for human activity recognition include statistical functions, time and/or frequency domain features, as well as heuristic features [9]. Each accelerometer data stream (AccX, AccY and AccZ) exhibits signal patterns that are distinctive for each of the arm movements, characterized by a set of features which are extracted from the signals [13]. In this investigation, we consider 10 time-domain features, extracted from each of the three individual sensor data streams as follows: 1) standard deviation - measure of the variability from the mean of the signal, 2) root mean square (rms) measure of the signal energy normalized by the number of samples, 3) information entropy - measure of the randomness of a signal [26], 4) jerk metric - rms value of the second derivative of the data normalized with respect to the maximum value of the first derivative [27], 5) peak number - obtained from gradient analysis of the signal, 6) maximum peak amplitude - measure of the amplitude of the peaks obtained after gradient analysis, 7) absolute difference - absolute difference between the maximum and the minimum value of a signal, 8) index of dispersion - ratio of variance to the mean, 
9) kurtosis - a measure of the 'peakedness' of a signal assuming a non-Gaussian distribution in the data, 10) skewness - a measure of the symmetry of the data assuming a non-Gaussian distribution in the data. Although the last two features are usually associated with defining the shape of a probability distribution, they can still be used as classifying features if they routinely return values that distinguish one pattern of data from another. Moreover, features such as jerk metric, peak number and maximum peak amplitude are reflective of the movement fluidity associated with assessing motor functionality in human limb [28]. Hence, we have a list of 30 features (3-axis $\times 10$ features) for each movement trial for each subject. The extracted features are linearly normalized and the best features for each subject are selected as discussed below.

\section{Feature selection}

The RELIEF algorithm [27], Clamping technique [16] and Principal Component Analysis (PCA) [11] are the most commonly used ranking/selection algorithms in the field of human activity recognition but are computationally intensive. We use the low-complexity class-separability measure based on scatter matrices to rank the 30 features. The scatter matrices quantify the scatter of feature vectors in the feature space. The rank of each individual feature for a multiple-class scenario is determined by the $R$ value as shown below [29]:

$$
R=\frac{S_{w}+S_{b}}{S_{w}}
$$

where, $S_{w}$ and $S_{b}$ are the within-class and between-class scatter matrices respectively. A high value of $R$ represents a small within-class variance and a large between-class distance among the data points in the respective feature space [29]. The ranked features are sorted in descending order with respect to their $R$ values. We employ a sequential forward selection $(S F S)$ technique, selecting the first $i$ features of the ranked feature set in each iteration $(i=2, \ldots, 30)$ and check if the data from the training phase can be correctly clustered in a multidimensional feature space as described in the next section.

\section{Cluster Formation}

The fundamental concept of cluster analysis is to form groups of similar objects as a means of distinguishing them from each other and can be applied in any discipline involving multivariate data [19]. With a given dataset $X=\left\{x_{i}\right\}, i=1, \ldots, n$ to be clustered into a set of $k$ clusters, the $k$-means algorithm iterates to minimize the squared error between the empirical mean of a cluster and the individual data points, defined as the cost function, $J$ :

$$
J(\theta, u)=\sum_{i=1}^{n} \sum_{j=1}^{k} u_{i j}\left\|x_{i}-\theta_{j}\right\|^{2}
$$

where, $\theta_{j}$ is the cluster center and $u_{i j}=1$ if $x_{i}$ lies close to $\theta_{j}$, or 0 if otherwise [30]. Initially $k$ centroids are defined and the data vectors are assigned to a cluster label depending on how close they are to each centroid. The $k$ centroids are recalculated from the newly defined clusters and the process of reassignment of each data vector to each new centroid is repeated. The algorithm iterates over this loop until the data vectors from the dataset $X$ form clusters and the cost function $J$ is minimized [29].

The Euclidean distance used to compute the squared distance between the vectors $x_{i}$ and the mean of each cluster $\theta_{j}$ has an undesirable effect of splitting large and elongated clusters, since most real datasets do not have a well-defined, isolated and spherical underlying cluster structure. By comparison, the use of the Mahalonobis distance which involves computing the covariance matrix of the data vector causes a large cluster to absorb nearby smaller clusters, leading to the creation of unusually large or small clusters. Hence we use the regularized Mahalonobis distance as mentioned in [30] which prevents the clustering algorithm from producing unusually large or small clusters.

$J\left(x_{i}, \theta_{j}\right)=\left(x_{i}-\theta_{j}\right)^{T}\left[(1-\lambda)\left(\sum_{j}+\varepsilon \mathrm{I}\right)^{-1}+\lambda \mathrm{I}\right]\left(x_{i}-\theta_{j}\right)$

where, $\Sigma_{j}$ is the covariance matrix of the $k$-th cluster and $I$ is the $d \times d$ identity matrix, $d$ is the input dimensionality (no. of feature vectors representing the data vector) and $\varepsilon$ is the regularization parameter. The value of $\lambda$ can be used as a parameter to control the choice of distance measure to be used, when $\lambda=0, J$ is the squared Mahalonobis distance and when $\lambda=1, J$ is the squared Euclidean distance.

In our exploration, we start with an initial value of $\lambda=1$ and after 3 iterations change it to $\lambda=0$. We run $k$-means clustering on the training data $[30$ features $\times 480$ movement trials $(240$ $A, 120-B, 120-C)]$ for each subject to form three clusters representing the three arm movements. The clustering algorithm runs on feature vectors which characterizes the 480 movement trials, sequentially selecting a combination of 2 to 30 ranked features in each run. We set a threshold of $25 \%$ of the expected number of data points for each cluster (i.e. $240 \pm$ 60 for Action A and $120 \pm 30$ for Action B and Action C) to check the number of data points in each of the three clusters formed. This threshold value was experimentally selected, as it produced the best results. If the number of data points in each cluster is within the threshold, we consider it as correctly clustered for each combination of features selected $(i=$ $2, \ldots, 30)$. We compute the distance of the mean of the training data for each class label from the cluster centroids for each feature combination and thereby assign each cluster with the class label that has its closest proximity to that particular class of training data.

\section{E. Distance Computation of Test Vector}

The data from the testing phase (80 test vectors - [ $\{(10$ of A) $+(5$ of B $)+(5$ of $C)\} \times 4$ trials $])$ for each subject is preprocessed and 30 features are extracted from each test vector. We then use a minimum distance classifier [29] to compute the distance of each test vector from the centroid of each 
cluster in a multi-dimensional feature space (considering only those feature combinations which resulted in successful clustering of the training data) based upon: a). Euclidean distance and b). Mahalonobis distance. The Mahalonobis distance takes into consideration the covariance of the clusters along with their mean for the maximum likelihood estimation of the covariance matrix and hence is effective for clusters with larger variance along one or many directions and in general having an ellipsoidal shape. The test vector is assigned to a particular cluster depending on the minimum distance computed for each of the two measures. The predicted label is further verified with respect to the annotations in the activitylist of Table I.

\section{RESULTS AND ANALYSIS}

The accuracy of recognizing the actions performed in the testing phase are presented in Table II, where we define recognition accuracy of an action as the number of times that action was correctly identified and assigned to the correct cluster, expressed as a percentage of the total number of actions in that class. We had in total 80 movement trials (actions) to be recognized ( 40 of $A, 20$ of $B, 20$ of $C$ ). The table also shows the minimum number of features that were required to successfully form the three clusters for each subject. The results in general, show that each subject required a different minimum number of features to successfully form 3 separate clusters from the training data, reflecting the variability in arm movement patterns between individuals. The right hand column in Table II shows the overall detection accuracy (total number of recognized actions expressed as a percentage of the total number of actions performed) for each subject, which covers the range $61 \%$ to $100 \%$ (average of $88 \%$ ) and $61 \%$ to $93 \%$ (average of $80 \%$ ) for the respective distance measures. We also present a ranked list of features in Table III (sorted in descending order) highlighting the features selected for each subject.

TABLE II. RECOGNITION ACCURACIES (\%) For EACH ARM MOVEMENT WiTH EUCLIDEAN (EUC) AND MAHALONOBIS (MAH) DiSTANCE ClASSIFIER.

\begin{tabular}{|c|c|c|c|c|c|c|c|c|c|}
\hline \multirow{3}{*}{ Subject } & \multirow{3}{*}{ Features } & \multicolumn{6}{|c|}{ Recognition accuracies } & \multirow{2}{*}{\multicolumn{2}{|c|}{$\begin{array}{l}\text { Overall } \\
\text { accuracy }\end{array}$}} \\
\hline & & \multicolumn{2}{|c|}{$A$} & \multicolumn{2}{|c|}{$B$} & \multicolumn{2}{|c|}{$C$} & & \\
\hline & & $E u c$ & Mah & Euc & Mah & Euc & Mah & Euc & Mah \\
\hline Subject1 & 11 & 100 & 98 & 100 & 100 & 100 & 75 & 100 & 93 \\
\hline Subject 2 & 2 & 80 & 75 & 5 & 5 & 80 & 95 & 61 & 61 \\
\hline Subject3 & 7 & 95 & 85 & 100 & 100 & 90 & 60 & 95 & 83 \\
\hline Subject4 & 23 & 95 & 63 & 100 & 100 & 85 & 100 & 94 & 81 \\
\hline
\end{tabular}

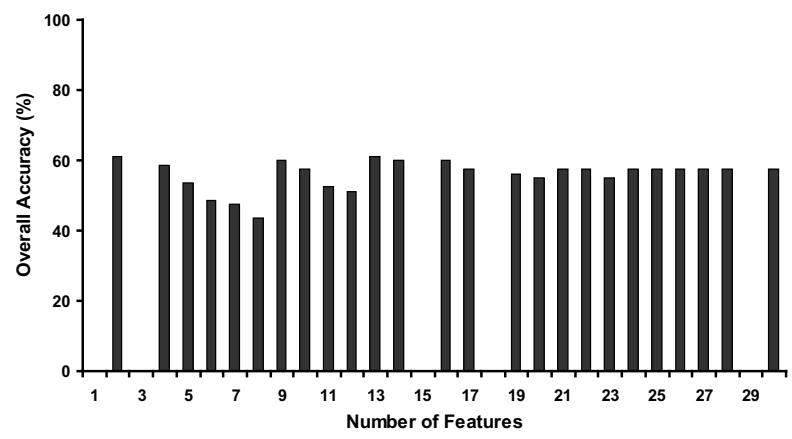

Fig 2. Change in accuracy with number of features selected (for Subject2)
TABLE III. FEATURES SELECTED From THEIR INDIVIDUAL RANKED ORDERS FOR EACH SUBJECT.

\begin{tabular}{|c|c|c|c|}
\hline Subject1 & Subject2 & Subject3 & Subject4 \\
\hline stddev_y & stddev_y & $r m s \_z$ & stddev_y \\
$r m s \_y$ & $r m s \_y$ & $r m s \_x$ & $r m s \_y$ \\
$r m s \_z$ & stddev_z & stddev_y & stddev_x \\
stddev_z & rms_x & stddev_x & $r m s \_x$ \\
rms_x & stddev_x & $r m s \_y$ & diff_y \\
diff_y & rms_z & entropy_z & max_mag_y \\
stddev_x & diff_x & stddev_z & diff_x \\
diff_z & max_mag_x & diff_y & max_mag_x \\
max_mag_y & diff_y & entropy_y & kurtosis_x \\
diff_x & skewness_y & kurtosis_x & kurtosis_z \\
max_mag_z & kurtosis_z & entropy_x & skewness_z \\
kurtosis_z & diff_z & max_mag_y & entropy_y \\
max_mag_x & skewness_z & diff_x & diff_z \\
entropy_z & max_mag_y & diff_z & max_mag_z \\
skewness_z & max_mag_z & peaks_z & kurtosis_y \\
kurtosis_x & kurtosis_y & skewness_y & stddev_z \\
entropy_x & entropy_z & peaks_x & entropy_x \\
skewness_y & skewness_x & max_mag_x & skewness_x \\
entropy_y & kurtosis_x & max_mag_z & peaks_y \\
kurtosis_y & entropy_x & skewness_x & skewness_y \\
skewness_x & entropy_y & kurtosis_z & entropy_z \\
peaks_x & peaks_z & kurtosis_y & rms_z \\
peaks_z & peaks_x & peaks_y & peaks_x \\
peaks_y & peaks_y & skewness_z & peaks_z \\
jerk_x & disp_y & jerk_z & jerk_z \\
disp_z & jerk_y & jerk_y & jerk_x \\
disp_y & jerk_x & disp_z & jerk_y \\
jerk_z & disp_z & jerk_x & disp_y \\
disp_x & disp_x & disp_x & disp_z \\
jerk_y & jerk_z & disp_y & disp_x \\
\hline
\end{tabular}

This shows that for each subject, the ranked order of features is different (reflecting on the different ways in which they perform a movement) and also shows the difference in the number of features required to form the clusters. In general we can consider the recognition accuracies achieved as being favorable, with the obvious exception of detecting Action B with Subject 2 with both the Euclidean and Mahalonobis minimum distance classifiers. It is worth noting that this subject required the smallest number of features to form clusters from the training data. This is somewhat counterintuitive - fewer features imply sufficient differences in arm movement patterns to make unique cluster formation easier. Whilst this may be the case, however, the low detection accuracy for Action $B$ could be accounted for by poor repeatability by the subject in this particular arm movement. The detection accuracies for Subject 2 using additional features are illustrated in Fig. 2, which reveals that increasing the number of features beyond 2 does not yield successful cluster formations or improved accuracy. In comparison with the results achieved, the minimum distance classifier based on Euclidean distance appears as the favorable choice of distance metric. This is favorable since computation of the Euclidean distance is far less complex than that of the Mahalonobis 
distance which involves the maximum likelihood estimation of the covariance matrix.

\section{DISCUSSION}

In this paper we describe a proof-of-concept methodology to recognize three fundamental movements of the upper limb typical of an activity of daily living, using only a wrist worn tri-axial accelerometer and partitional $k$-means clustering algorithm to form 3 pre-defined clusters with the training data. Test data are then associated to each of these clusters using a minimum distance classifier based on Euclidean and Mahalonobis distance. Our results show that we can detect all three arm movements performed in a real world scenario with an accuracy of $61-100 \%$ using between 2-23 time domain features and both the Euclidean distance and the Mahalonobis distance based classifiers. Furthermore, our methodology exhibits an overall average accuracy of $88 \%$ across all subjects and arm movement types using the Euclidean distance metric where the number of features used is subject-specific, reflecting the variability inherent in human movement. The methodology will be scalable and adaptable when applied to a larger group of subjects as it looks for the best combination of features to achieve the highest overall accuracy for each individual subject which when applied in the field of remote health monitoring will provide a gross measure of subjectspecific rehabilitation. We believe that this methodology can be used for real time detection of arm movements where the cluster formation and feature selection can be performed offline whereas computation of the selected features and distance from cluster centroids can be performed in an online mode thereby providing energy efficient solutions for bodyworn sensors: an essential component of telehealth systems. The study can be extended further to observe the effects of other sensors and arm attachment locations on a larger sample population with appropriate cross-validation techniques applied to the cluster formation on the training data.

\section{REFERENCES}

[1] B. Najafi et al., "Ambulatory system for human motion analysis using a kinematic sensor: Monitoring of daily physical activity in the elderly," IEEE Trans. Biomed. Eng., vol. 50, no.6, pp. 711-723, Jun. 2003.

[2] A. Hadjidj, A. Bouabdallah, and Y. Challal, "Rehabilitation supervision using wireless sensor networks," in Proc. IEEE WoWMoM, Luca, Italy, pp.1-3, Jun. 2011.

[3] M.S. Raisinghania, A. Benoit and J. Ding, "Ambient intelligence: Changing forms of human-computer interaction and their social implications," Texas Digital Library, vol. 5, no.4, 2004.

[4] D. Merrill, J. Kalanithi and P. Maes, "Siftables: Towards sensor network user interfaces," in Proc 1st Int. Conf. on Tangible and Embedded Interaction, Baton Rouge, LA, pp. 75-78, Feb. 2007.

[5] S. Armstrong, "Wireless connectivity for health and sports monitoring: a review," B. J. Sports Medicine, vol. 41, pp. 285-289, Jan. 2007.

[6] M. Ermes et al., "Detection of daily activities and sports with wearable sensors in controlled and uncontrolled conditions," IEEE Trans. Inf. Technol. Biomed., vol. 12, no.1, pp. 20-26, Jan. 2008.

[7] F.E. Martınez-Pérez et al., "Activity inference for ambient intelligence through handling artifacts in a healthcare environment," Sensors, vol. 12, no.1, pp. 1072-1099, Jan. 2012.

[8] J. Parkka et al., "Activity classification using realistic data from wearable sensors," IEEE Trans. Inf. Technol. Biomed., vol.10, no.1, pp.119-128, Jan. 2006
[9] K. Altun, B. Barshan, O. Tuncel, "Comparative study on classifying human activities with miniature inertial and magnetic sensors", Pattern Recognition, vol. 43, no. 10, pp. 3605-3620, Oct. 2010.

[10] C. Zhu and W. Sheng, "Motion- and location-based online human daily activity recognition," Pervasive and Mobile Computing, vol. 7, no. 2, pp. 256-269, Apr. 2011.

[11] L.Wang et al., "A hierarchical approach to real-time activity recognition in body sensor networks", Pervasive and Mobile Computing, vol. 8, no. 1, pp. 115-130, Feb. 2012.

[12] P. Turaga et al., "Machine recognition of human activities", IEEE Trans. Circ. Syst. Video Technol., vol. 18, no. 11, pp. 1473-1488, Nov. 2008.

[13] O. Banos et al., "Daily living activity recognition based on statistical feature quality group selection," Expert Systems with Applications, vol. 39, no. 9, pp. 8013-8021, Jul. 2012.

[14] A. Fleury, M. Vacher, and N. Noury, "SVM-based multimodal classification of activities of daily living in health smart homes: Sensors, algorithms, and first experimental results," IEEE Trans. Inf. Technol. Biomed., vol.14, pp. 274-283, Mar. 2010.

[15] D. Fuentes et al., "Online motion recognition using an accelerometer in a mobile device," Expert Systems with Applications, vol. 39, no. 3, pp. 2461-2465, Feb. 2012.

[16] S. Chernbumroong et al., "Elderly activities recognition and classification for applications in assisted living," Expert Systems with Applications, vol. 40, no. 5, pp. 1662-1674, Apr. 2013.

[17] H. Junker et al., "Gesture spotting with body-worn inertial sensors to detect user activities," Pattern Recognition, vol. 41, no. 6, pp. 20102024, Jun. 2008.

[18] N. Bicocchi, M. Mamei, F. Zambonelli, "Detecting activities from bodyworn acclerometers via instance-based algorithms", Pervasive and Mobile Computing, vol. 6, no. 4, pp. 482-495, Aug. 2010.

[19] T.Warren Liao, "Clustering of time series data-a survey", Pattern Recognition, vol. 38, no. 11, pp. 1857-1874, Nov. 2005.

[20] L. Bao and S. Intille, "Activity recognition from user-annotated acceleration data", in Proc. $2^{\text {nd }}$ Int. Conf. Pervasive Comput, pp. 1-17, 2004.

[21] A. Burns et al., "Shimmer - A wireless sensor platform for noninvasive biomedical research," IEEE Sensors Journal, vol. 10, no. 9, pp.15271534, Sep. 2010.

[22] C. Kendell and E.D. Lemaire, "Effect of mobility devices on orientation sensors that contain magnetometers," J. Rehab. Res. Dev., vol. 46, no. 7, pp. 957-962, 2009.

[23] S. L. Wolf et al., "The Excite trial: relationship of intensity of constraint induced movement therapy to improvement in the wolf motor function test," Restorative Neurology and Neuroscience, vol. 25, no. 5, pp. 549562, Mar. 2007.

[24] S. L. Wolf et al., "Pilot normative database for the wolf motor function test," Archives of Physical Medicine and Rehabilitation, vol. 87, no. 3, pp. 443-445, Mar. 2006.

[25] D. M. Morris et al., "The reliability of the Wolf Motor Function Test for assessing upper extremity function after stroke," Archives of Physical Medicine and Rehabilitation, vol. 82, no. 6, pp. 750-755, Jun. 2001.

[26] I. Kim et al., "ADL classification using triaxial accelerometers and RFID," in Proc. First Int. Conf. Ubiquitous Computing Convergence Technology, 2007.

[27] S. Patel et al., "A novel approach to monitor rehabilitation outcomes in stroke survivors using wearable technology," Proceedings of the IEEE, vol. 98, no. 3, pp. 450-461, Mar. 2010.

[28] B. Rohrer, S. Fasoli, et al, "Movement smoothness changes during stroke recovery," The Journal of Neuroscience, vol. 22, pp. 8297-8304, Sep. 2002.

[29] S. Theodoridis and K. Koutroumbas, "Pattern Recognition", 4th ed., Elsevier, pp. 30-31 and pp. 280-288, 2008.

[30] J. Mao, A.K. Jain, "A self-organizing network for hyperellipsoidal clustering (HEC)", IEEE Trans. Neural Networks, vol. 7, no.1, pp. 1629, Jan. 1996. 